\title{
ÍNDICE DE MATERIAS
}

Páglass

Advertencias preliminares $\ldots \ldots \ldots \ldots \ldots \ldots \ldots \ldots$

\section{TABLAS}

Indice

marginal

P. at. I .... Pesos atómicos de los elementos y logaritmos res$\begin{array}{llllllllllll}\text { pectivos } & \ldots & \ldots & \ldots & \ldots & \ldots & \ldots & \ldots & \ldots & \ldots & \ldots & \ldots\end{array}$

P. ml. II .... Pesos y logari:mos respectivos de los átomos, grupos atómicos, moléculas y equivalentes más frecuentemente usados (así como múltiplos infe-

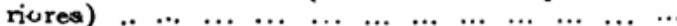

III ... Múltiplos superiores de algunos pesos atómicos y moleculares con logaritmos respectivos... .......

P. eq. IV ... A. Pesos equivalentes volumétricos y logaritmos

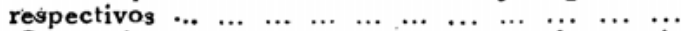

B. Correcciones para tener en cuenta el empuje del aire en las pesadas exactas ... ... ... ... ...

C. Pesos equivalentes volumétricos y logaritmos reopectivos (pesos detyerminados en el aire) ...

F. anal V .... "Factores" analíticos y esiequiométricos con sus

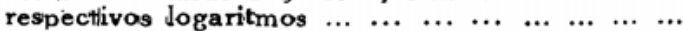

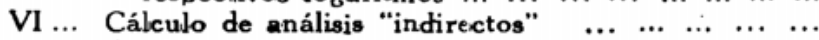

Vla . Análisis crioscópico, según lbing-Ebert ... ... ...

VII . Determinación volumétrica del nitrógeno y otros gases. - Tabla de reducción de gases .........

VIla Corrección barométrica (en Torr) $\ldots \ldots \ldots c c c c 114$

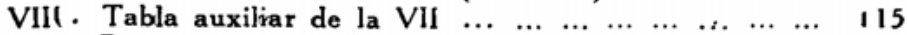

IX.. Determinación valumétrica de los gases más im$\begin{array}{llllllllllll}\text { portantes } & \ldots & \ldots & \ldots & \ldots & \ldots & \ldots & \ldots & \ldots & \ldots & \ldots & \ldots\end{array}$

$\mathrm{X}$.... Determinación volumétrica de substancias que des.

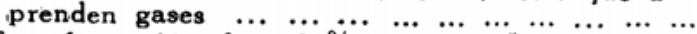

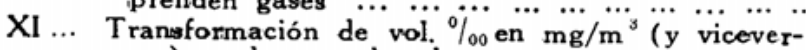

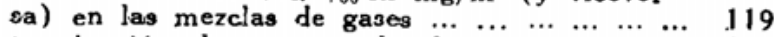

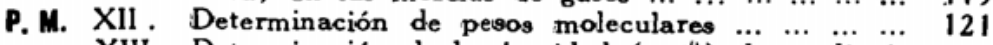

XIII Determinación de la densidad $\left(\varphi t^{*}\right)$ de un liquido por pesada en el aire) $\ldots \ldots \ldots \ldots \ldots \ldots \ldots \ldots$

XIV Densidad del rqua $(\underline{v} w)$ a distintas temperaturas $t$ $\left({ }^{\circ} \mathrm{C}\right)$ y logaritmos respectivos $\ldots \ldots \ldots \ldots \ldots$

XV. Determinación gravimétrica de volúmenes $\ldots \ldots \ldots . . . .123$

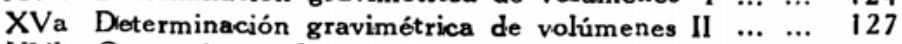

XVI Correcciones de temperaturas para el análisis vo$\begin{array}{lllllllllllll}\text { lumétrico } & \ldots & \ldots & \ldots & \ldots & \ldots & \ldots & \ldots & \ldots & \ldots & \ldots & \ldots & 129\end{array}$

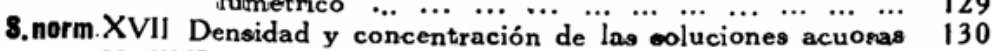

XVIll Temperatura y densidad del mercurio $\ldots . . . \quad \ldots . . . \quad 136$ 
XIX.... Logaritmos de los valores $\frac{n^{2}-1}{n^{2}+2}$

XX...... Solubilidad a $20^{\circ}$ de algunas substancias impor-

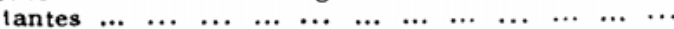

$\lambda X I$.... Puente de Wheatstione. Logaritmos de los valores de $a:(1.000 a)$ para variaciones de $a$ de 1

Eiec. XXII... Electroquímica. $\begin{array}{lllllllllllllll}\text { a } 999 & \ldots & \ldots & \ldots & \ldots & \ldots & \ldots & \ldots & \ldots & \ldots & \ldots & \ldots & \ldots & 140\end{array}$

A. Equivalentes electroquímicos. Pilas normales . 142

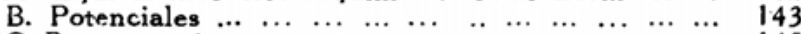

$\begin{array}{llllllllllllll}\text { C. Batmometría } & \ldots & \ldots & \ldots & \ldots & \ldots & \ldots & \ldots & \ldots & \ldots & \ldots & \ldots & \ldots & 145\end{array}$

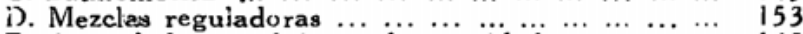

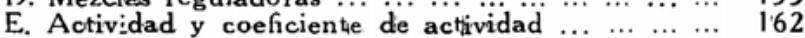

Ind. XXIII.. Indicadores, batmometría óptica, colorimetría.

A. Recopilación de algunos ind:cadoresi importantes 164

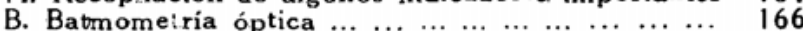

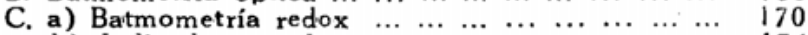

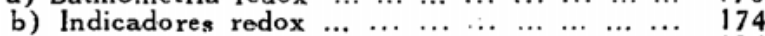

$\begin{array}{llllllllllll}\text { D. Colorimetría } & \ldots & \ldots & \ldots & \ldots & \ldots & \ldots & \ldots & \ldots & \ldots & \ldots & 175\end{array}$

Term. XXIV.. Termoquimica.

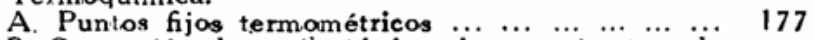

B. Corrección de capilaridad en los termómetros de

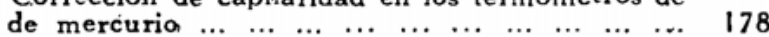

C. Paso de grados Fahrenheit ( $\left.{ }^{\circ} \mathrm{F}\right)$ a grados $\mathrm{Ce}$ I-

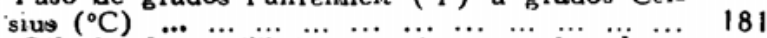

D. Cálculo de equilibrios quimicos con los datos

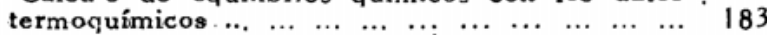

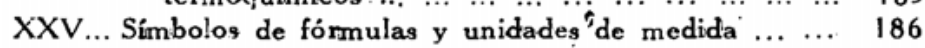

Unid. XXVI.. Unidades, constantes y magnitudes de conversión

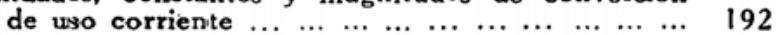

XXVII. Unidades de medidas anticuadas... $\ldots \ldots \ldots \ldots \ldots \ldots$

XXVIIl Nucva nomenclatura de los compuestos inorgánicos 198

$\begin{array}{lllllllllll}\text { XXIX.. Cálculo de errores } & \ldots & \ldots & \ldots & \ldots & \ldots & \ldots & \ldots & \ldots & \ldots & 202\end{array}$

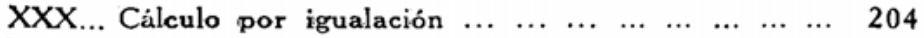

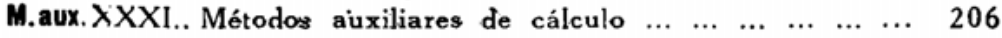

XXXIl. Evaluación de las radiografías
$\ldots$

$\begin{array}{lllllllllllllll}\text { Notas } & \ldots & \ldots & \ldots & \ldots & \ldots & \ldots & \ldots & \ldots & \ldots & \ldots & \ldots & \ldots & \ldots & 224\end{array}$

Aclar.

Actaraciones

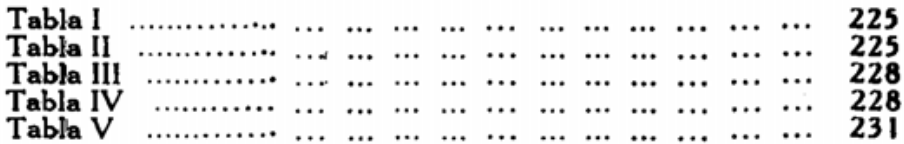


Indice

\section{Aclaraciones}

$\begin{array}{llllllllllllll}\text { Tabla.VI } & \ldots \ldots \ldots \ldots \ldots & \ldots & \ldots & \ldots & \ldots & \ldots & \ldots & \ldots & \ldots & \ldots & \ldots & \ldots & 235\end{array}$

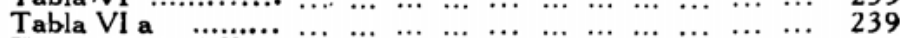

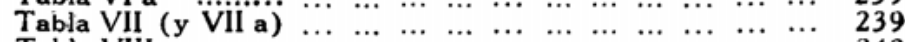

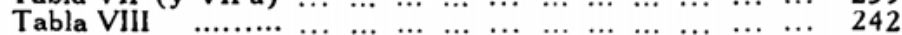

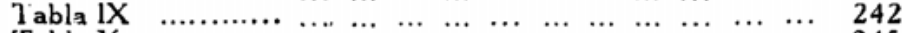

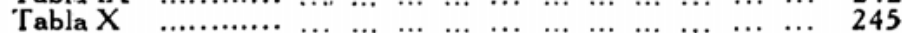

$\begin{array}{llllllllllllllll}\text { Tabla XI } & \ldots & \ldots \ldots \ldots & \ldots & \ldots & \ldots & \ldots & \ldots & \ldots & \ldots & \ldots & \ldots & \ldots & \ldots & 246\end{array}$

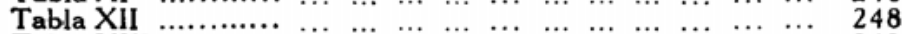

$\begin{array}{llllllllllllll}\text { Tabla XIII } & \ldots \ldots \ldots \ldots . & \ldots & \ldots & \ldots & \ldots & \ldots & \ldots & \ldots & \ldots & \ldots & \ldots & \ldots & \ldots \\ \end{array}$

Tabla XIV

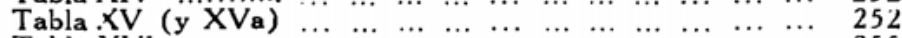

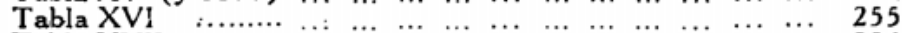

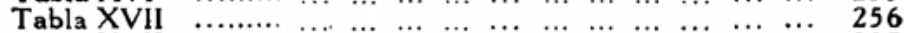

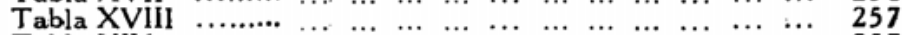

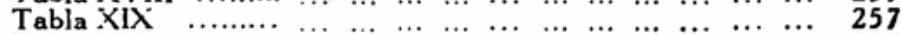

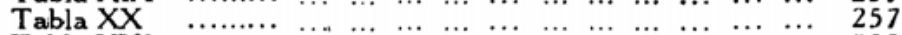

$\begin{array}{lllllllllllllll}\text { Tabla XX1 } & \ldots \ldots \ldots & \ldots & \ldots & \ldots & \ldots & \ldots & \ldots & \ldots & \ldots & \ldots & \ldots & \ldots & \ldots & 258\end{array}$

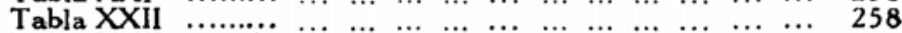

$\begin{array}{llllllllllllll}\text { Tabla XXIII } & \ldots \ldots \ldots \ldots & \ldots & \ldots & \ldots & \ldots & \ldots & \ldots & \ldots & \ldots & \ldots & \ldots & \ldots & 258\end{array}$

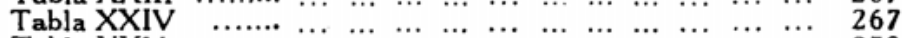

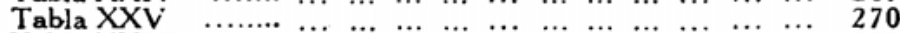

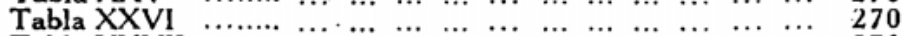

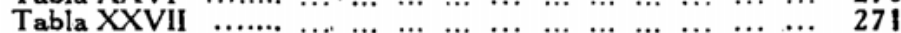

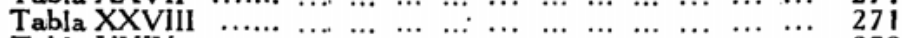

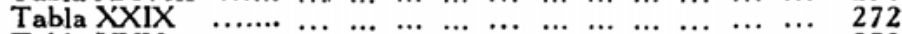

$\begin{array}{llllllllllllll}\text { Tabla XXX } & \ldots \ldots \ldots & \ldots & \ldots & \ldots & \ldots & \ldots & \ldots & \ldots & \ldots & \ldots & \ldots & \ldots & 272 \\ & \ldots & \ldots & \ldots & \ldots & \ldots & \ldots & \ldots & \ldots & \ldots & \ldots & \ldots & 273\end{array}$

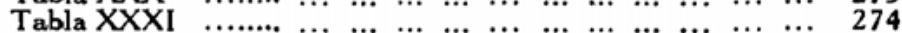

$\begin{array}{llllllllllllll}\text { Tabla XXXII } & \ldots & \ldots & \ldots & \ldots & \ldots & \ldots & \ldots & \ldots & \ldots & \ldots & \ldots & \ldots & \ldots \\ \end{array}$

Mant. Mantigas de cinco cifras de los logaritmos decimaleis de todos los números de cuatro cifras comprendidos entre 1.000 y 9.999, con las partes proporcionales corres-

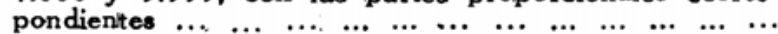

Mantisas de cuatro cifras de los logaritmos decimales de todos los números de tres cifras comprendidos entre 100 y 999, y mantisas de cinco cifras correspondientes a todos los números de cautro cifras comprendidos entre 1.000 y $2.000 \ldots \ldots \ldots \ldots$

$\begin{array}{llllllllllllll}\text { Indice alfabético } & \ldots & \ldots & \ldots & \ldots & \ldots & \ldots & \ldots & \ldots & \ldots & \ldots & \ldots & \ldots & 307\end{array}$ 